\title{
Sequence analysis of 16S rRNA gene in Rhinosporidium seeberi shows similarity to plant chloroplast DNA
}

\author{
Karvita B Ahluwalia $^{1 *}$, Dhara B Dhaulakhandi ${ }^{1}$, Lalit C Garg ${ }^{2}$ \\ ${ }^{1}$ Cell Biology and Electron Microscope Section, Department of Biophysics, All India Institute of Medical Sciences, New Delhi - 110029, India; ${ }^{2}$ Gene \\ Regulation Laboratory, National Institute of Immunology, New Delhi - 110067, India; Karvita B Ahluwalia, E-mail: karvitaa@yahoo.com , Tel.: +91 11 \\ 26535914; *Corresponding author.
}

Received June 28, 2010; Accepted August 26, 2010; Published September 20, 2010

\begin{abstract}
:
Identity of causative agent of rhinosporidiosis (Rhinosporidium seeberi ) has been controversial since the disease was described in $1900 . \quad$ Extensive sequence alignments and phylogenetic analyses of 16S rRNA gene detected recently by us in $R$. seeberi , revealed $99 \%$ similarity with $16 \mathrm{~S}$ rDNA in chloroplasts of flowering plants. Study demonstrates $R$. seeberi is a pigmented prokaryote displaying some characteristics of cyanobacteria, and contains 16S rDNA present in chloroplasts of all groups of land plants. This study and our recent publication of 2006 are the first molecular studies using purified organismal DNA extracted from $R$. seeberi free of infected tissue.
\end{abstract}

Key words: Rhinosporidium seeberi ; 16S rRNA gene; sequence analysis; chloroplast DNA; molecular phylogeny.

Abbreviation: RB - Round body

\section{Background:}

Rhinosporidiosis produces polypoid masses in nose and other sites in individuals exposed to fresh water ponds. An outbreak of rhinosporidiosis occurred among people on holiday near Silver Lake in Serbia [1]. The disease has worldwide distribution and is endemic in southern India, Sri Lanka and Argentina. Rhinosporidium seeberi is spherical upto 300- 400 $\mu \mathrm{m}$ in diameter, visible in histological sections as a round body (RB) filled with endospores that are discharged and enlarge into new RBs. All in vivo stages in $R$. seeberi 's life cycle (enlargement, maturation of round bodies and release of endospores) have been demonstrated in vitro using monolayers of a human rectal tumor cell line [2]. Immunological defense mechanism is not known, only phagocytosis is reported [3]. First described by Seeber in 1900 [4], taxonomy of $R$. seeberi has been debated throughout the century. No treatment is effective; multiple recurrences are common after surgery. The disease is not transmitted to family members of affected individuals. RBs have not been seen in natural habitats, nor in pond water samples from 3 endemic regions in India [5].

Recently we detected a 16S rRNA gene (GenBank accession no. AJ440719) in purified RBs carefully isolated from infected tissue, and determined the sequence [6]. This study is a detailed analysis of sequence of $16 \mathrm{~S}$ rRNA gene.

\section{Methodologies:}

Individual uncontaminated RBs $(R$. seeberi ) were isolated from infected tissue (Supplementary Figure 1) and pure organismal DNA extracted from a single RB (details in [6]). Briefly, between 15-25 ng DNA extracted from a single RB was checked for purity spectrophotometrically and by running on $0.85 \%$ agarose. Experiments were repeated 3 times using 3 different clinical samples. PCR primers identical to those used in a previous study [7] were procured from Yale University, Connecticut. Gene Amp 2400 PCR system (Perkin Elmer) was used. Clustal algorithm was used for multiple sequence alignment in LASERGENE software (DNA Star). Phylogenetic trees were constructed using Saitou \& Nei’s [8] neighbor-joining method.

\section{Results and Discussion:}

$R$. seeberi 's 1458 bp 16S rRNA gene sequence (AJ440719) aligned in BLASTN with 16S rDNA sequences in GenBank (no domain specified) showed high similarity (99\%) with DNA in chloroplasts (cpDNA) of flowering plants Solanum tuberosum, Nicotiana tabacum, $N$. plumbaginifolia, Arabidopsis thaliana, Carica papaya and Vitis vinifera. cpDNA in 70 genera of higher plants, liverwort Marchantia, green alga Chara, Spirogyra and related genera displayed 95-97\% identity with AJ440719. Oryza sativa showed $96 \%$ identity with cpDNA as also with genomic DNA in chromosome 10. cpDNA in nonphotosynthetic alga Prototheca and amoeba Paulinella chromatophora showed $82 \%$ and $84 \%$ identity, respectively. BLAST search specifying domain bacteria indicated high similarity with many cyanobacteria. Phylogenetic tree (Figure 1) shows $R$. seeberi 's sequence positioned unambiguously among cpDNA of flowering plants. AJ440719 clusters with cpDNA with high bootstrap support. Chloroplast genes in human apicomplexan pathogens are not phylogenetically close to $R$. seeberi 's sequence (Supplementary Figure 2).

Pairwise alignment of 1458 bp sequence (AJ440719) in BLAST2 with 16S rDNA in chloroplasts of several plants indicates most differences with $R$. seeberi 's sequence in nucleotide positions 1 to 82 . Thus, cpDNA in Solanum nigrum displayed 99\% identity with nucleotides 79-1458 with 4 gaps, and identity of $93 \%$ for nucleotides $4-63$ with no gap. Two gene fragments, 1-82 and 83-1458 were aligned separately in BLAST2 in 2 steps. Nucleotides 1- 82 aligned mostly with bacteria including 14 proteobacteria and 5 pathogenic bacteria (identity 90-95\%), nucleotides 
83-1458 showed 95-99\% alignment with cpDNA of numerous plants $\mathrm{G}+\mathrm{C}$ content of AJ440719 is 56.8 .

Chloroplast sequence in $R$. seeberi displayed cyanobacterial sequence characteristics. Two palindromic repeats and sequence signature (AATTTTCCG) of cyanobacteria were found in RB's 16S rRNA gene [6]. In this study, multiple sequence alignments showed that sequence signature is shared with $16 \mathrm{~S}$ rDNA in 11 cyanobacteria and cpDNA in plant Carica papaya, and flanking nucleotides are also similar (Supplementary Figure 3). Among 826 conserved nucleotide positions in AJ440719, 773 (92.4\%) are shared with 11 cyanobacteria and cpDNA in Carica, while conserved nucleotides in segment 116-225 are dissimilar. The single conserved nucleotide $\mathrm{T}$ is in position 799 , and $\mathrm{A}$ is in position 1233 as reported in cyanobacteria [9]. Pairwise alignment of 1458 bp sequence (AJ440719) in BLAST2 with several cyanobacteria showed identity range of $79-86 \%$, with most differences in first segment of 82 nucleotides. Low identity of $79-86 \%$ may be result of comparison with chloroplast-like 16S rRNA sequence in RB. Another different copy of 16S rRNA gene, that is not chloroplast-like, could possibly be present in $R$. seeberi 's genome. Different $16 \mathrm{~S}$ rRNA sequences could be present in a single cell genome $[\mathbf{1 0}, \mathbf{1 1}]$.

\section{Taxonomic Placement of $R$. seeberi}

Ashworth [12] considered organism in rhinosporidiosis to be a fungal sporangium filled with spores, and named it Rhinosporidium seeberi Mycology textbooks describe $R$. seeberi as a fungus of uncertain taxonomy. We used fungal-specific primers for 18S and 28S rRNA genes, no amplification was seen with DNA from RBs as template, negating fungus in $R$. seeberi [6]. Presence of chlorophyll led Vanbreuseghem [13] to suggest $\mathrm{RB}$ as an alga that was refuted by some authors [3] as chloroplasts have not been seen in RB. Photosynthetic pigments, also found by us [14], rule out fungus.

The in vivo life cycle of $R$. seeberi is initiated with the entry of endospores into host epithelium $[\mathbf{1 5}, \mathbf{1 6}]$. Study of pond water samples revealed striking similarity between mature endospores in RB and cyanobacterium Microcystis [5]. PCR using genomic DNA of $R$. seeberi showed a sequence that is strongly amplified by $16 \mathrm{~S}$ rRNA primers specific for cyanobacterium Microcystis [6]. For a pathogen as little understood as $R$. seeberi , accurate assessment of ultrastructure could complement molecular data. Our findings, and of others indicate RBs phenotypic relationship with cyanobacteria. These include dispersed chromatin [15-17], polyhedral bodies, wall peptidoglycan [5], photosynthetic pigments $[\mathbf{1 3}, \mathbf{1 4}]$, and red auto fluorescence excited with green (510-540 nm) wavelengths (this study) that is typical of photosynthetic pigments [18].

Another study in two laboratories, based on DNA from infected tissue, proposed $R$. seeberi 's relationship with the protist Dermocystidium [7, 19]. Since rhinosporidiosis develops in individuals exposed to fresh water ponds, the presence of aquatic protists like Dermocystidium in infected tissue is not ruled out. Cysts and spores of Dermocystidium sloughed from gills of fish "could be a significant source of infection in fresh water systems” [20]. Even a low number of Dermocystidium spores in infected tissue could be detected by the sensitive PCR. Authors in both laboratories selected 18S SSU rRNA primers F1-fw F2-rev and NS1 NS8 (degenerated) for fungi that also anneal to some protists $[\mathbf{1 9}, \mathbf{7 ]}$. Therefore, DNA of Dermocystidium, if present in infected tissue, would be amplifiable with these primers. Although primer F2-rev differs from the sequence in 2 nucleotide positions and primer F3-rev differs in 3 nucleotide positions, these primers gave amplification, "data not shown" [19]. Primer Rhino-fw differs from the sequence at 4 nucleotide positions and Rhino-rev differs in 2 nucleotide positions, they are reported to give amplification [19]. In one study [19], since control samples of nasal polyps from patients without rhinosporidiosis came from humans not exposed to fresh water ponds, the samples are not expected to harbor aquatic protists, and this explains why the authors [19] got negative results with PCR. Controls are lacking in the other study [7]. We procured the same 18S SSU rRNA primers mentioned in their study, for using with organismal DNA extracted from purified RBs, PCR did not show amplification with those primers. As 16S rRNA sequence can be compared with 18S rRNA [10], a comparison made between $R$. seeberi 's sequence AJ440719 and 18S rDNA in [19] did not show similarity.

\section{Conclusion:}

$R$. seeberi is a pigmented prokaryote with cyanobacterial features, and contains $16 \mathrm{~S}$ rDNA identical to that in chloroplasts of land plants. This is the first report of a chloroplast sequence in $R$. seeberi , and since humans do not harbor chloroplast, can be used as target for development of chemotherapy. Detection of a sequence similar to AJ440719 in a cyanobacterial genus, or species/strain of Microcystis, might be useful for taxonomic identification of RB. DNA from RB generated a strong band with primers for Cyanobacterium microcystis [6]. Taking a cue from this observation, the presence of cyanobacterium-specific repeat sequences and sequence signature found in the sequence of $R$. seeberi 's chloroplast-like 16S rRNA gene [6] should now be searched elsewhere in the genome. Sequencing the entire genome of $R$. seeberi would be rewarding.

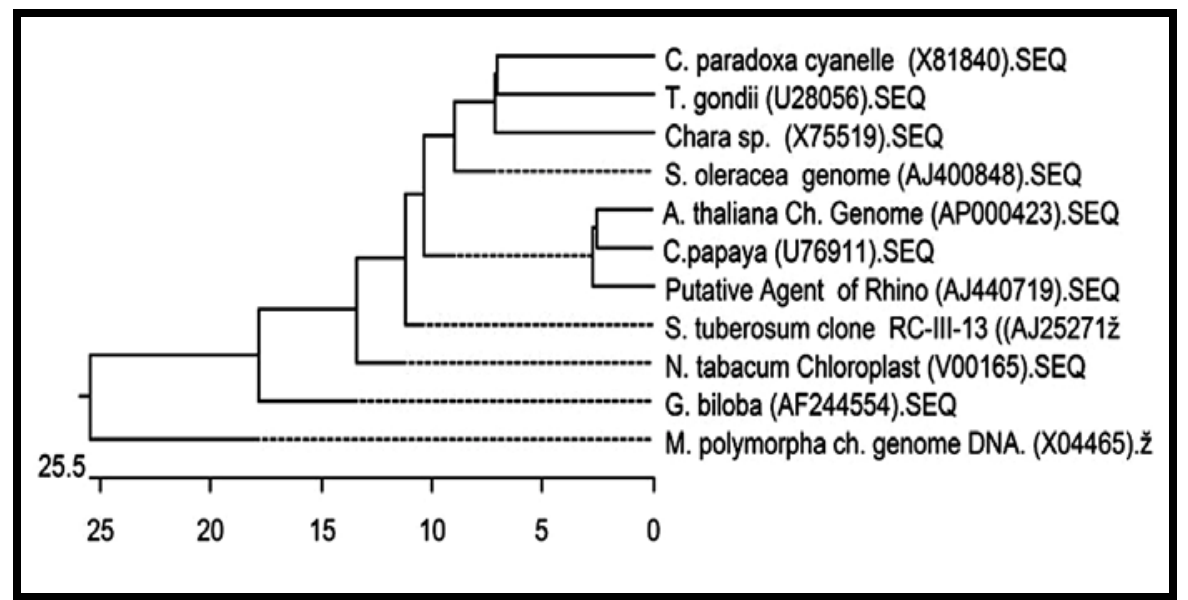

Figure 1: Phylogenetic tree showing affinities of $R$. seeberi 's sequence (AJ440719) with chloroplast DNA in genera from different taxonomic groups. Flowering plants: Arabidopsis thaliana, Carica papaya, Solanum tuberosum, S. oleracea, Nicotiana tabacum. Gymnosperm: Ginkgo biloba. Bryophyte: Marchantia polymorpha. Streptophyte: Chara. Cyanelle of Cyanophora paradoxa. Human pathogen: Toxoplasma gondii. 


\section{Acknowledgements:}

K.B.A. thanks Prem Chopra, Professor of Pathology, AIIMS for useful discussions. Department of Science and Technology, Government of India and All India Institute of Medical Sciences provided research grants.

\section{References:}

[1] Z Vukovic et al. J. Trop. Med. Hyg. 98:333 (1995) [PMID: 756326 2]

[2] MG Levy et al. Science. 234: 474 (1986) [PMID: 3764422]

[3] M Thianprasit et al. Curr. Topics Med. Mycol. 5:64-85 (1989) [PMID: 2688923]

[4] GR Seeber (1900) Thesis, Universite Nacionale de Buenos Aires

[5] KB Ahluwalia et al. Amer. J. Rhinol. 11: 479 (1997) [PMID: 9438062]

[6] DB Dhaulakhandi et al. Infec. Genetics Evol. 6: 331 (2006) [PMID: 16418014]

[7] RA Herr et al. J. Clin. Microbiol. 37: 2750 (1999) [PMID: 10449446]
[8] N Saitou et al. Mol. Biol. Evol. 4:406 (1987) [PMID: 3447015]

[9] CR Woese. Microbiol. Rev. 51: 221 (1987) [PMID: 2439888]

[10] JE III Clarridge. Clin. Microbiol. Rev. 17: 840 (2004) [PMID: 15489351]

[11] J Viezens et al. Microbiology154: 2881 (2008) [PMID: 18759822]

[12] JH Ashworth, Trans. Roy. Soc. Edinburgh (1923) 2: 301.

[13] R Vanbreuseghem. Int. J. Dermatol. 12: 20 (1973) [PMID: 4571604]

[14] KB Ahluwalia. J. Laryngol. Otol. 113: 513 (1999) [PMID: 10605581]

[15] M Kannan-Kutty et al. Pathology 6: 63 (1974) [PMID: 4423221]

[16] DF Savino et al. Ophthalmology 90: 1482(1983) [PMID: 6677849]

[17] FA Kennedy et al. J. Med. Vet. Mycol. 33: 157 (1995) [PMID: 7666295]

[18] JW Hofstraat et al. J. Plankton Res. 16: 1197. (1994)

[19] DN Fredricks et al., Emerg. Infec. Dis. 6: 273 (2000) [PMID: 10827117]

[20] RE Olson et al. Dis. Aquatic Organisms 12: 41 (1991)

Edited by P. Kangueane

Citation: Ahluwalia et al. Bioinformation 5(3): 89-96 (2010) License statement: This is an open-access article, which permits unrestricted use, distribution, and reproduction in any medium, for non-commercial purposes, provided the original author and source are credited. 


\section{Bioinformation}

Volume 5

\section{Supplementary material:}

Supplementary Figure 1: Isolation of single round bodies. Purified single round bodies after isolation from infected tissue in Eppendorf tube.

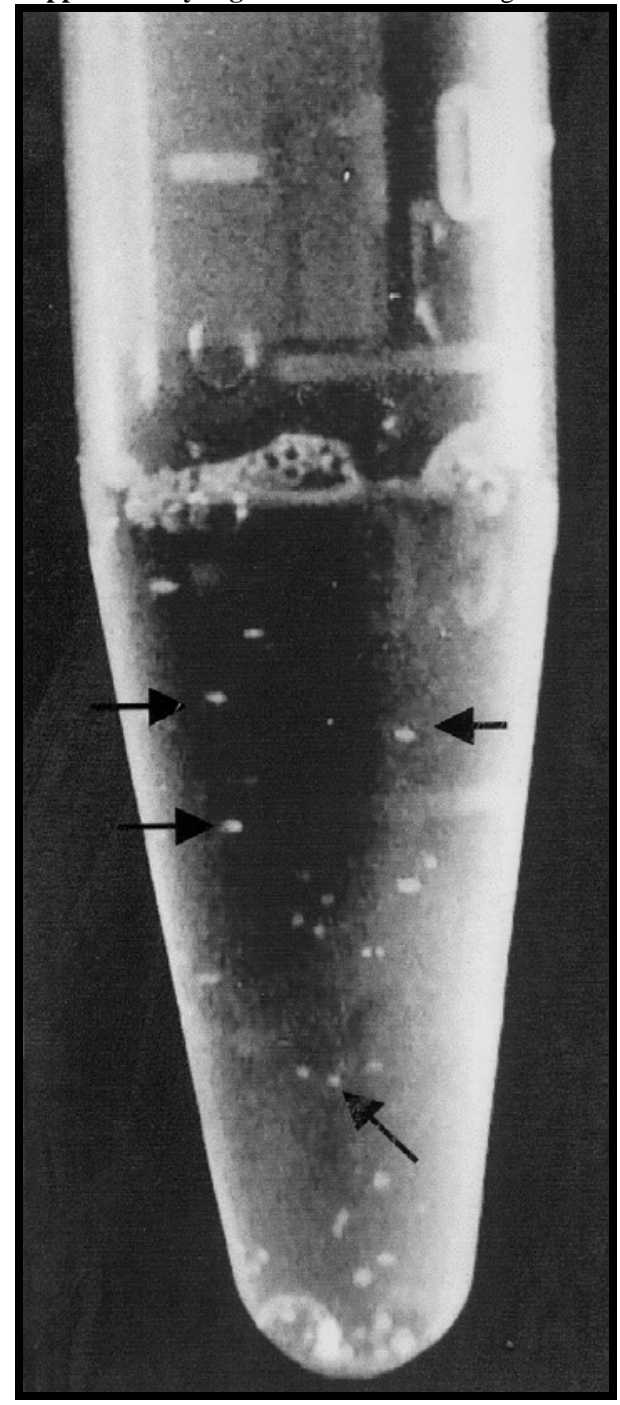


Supplementary Figure 2: Extended phylogenetic tree showing affinities of $R$. seeberi 's chloroplast sequence (AJ440719) with relevant taxa. AJ440719 clusters with chloroplast DNA in flowering plants. Tree also shows 16S rDNA in cyanobacteria, and cpDNA in amoeba Paulinella chromatophora, cyanelle of Cyanophora, nonphotosynthetic Prototheca and Polytoma, as well as cpDNA in apicomplexan pathogens (Toxoplasma gondii, Sarcocystis, Eimeria, Babesia). The mesomytcetozoans (Dermocystidium, Ichthyophonus) and fungi (Chytriomyces,

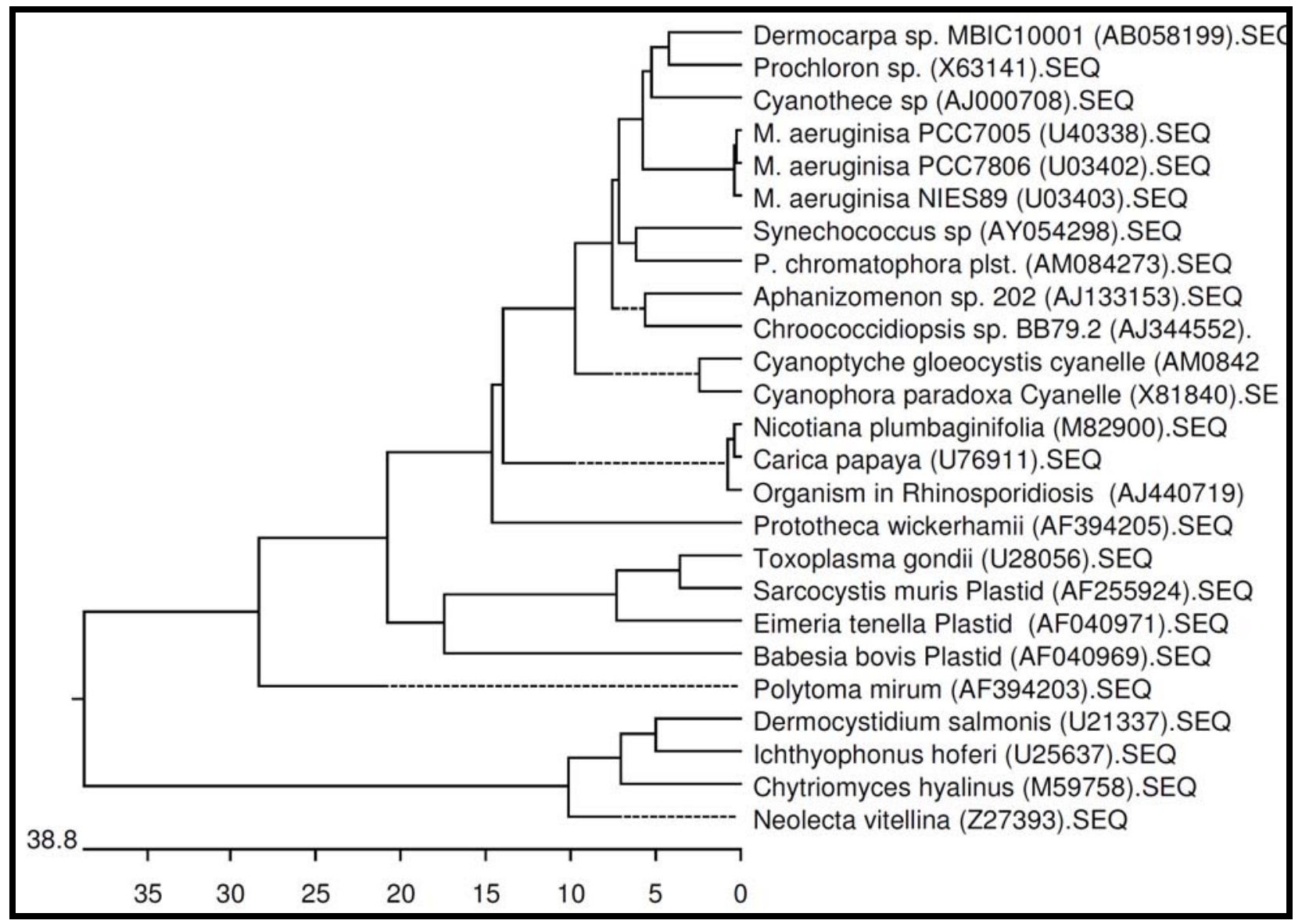


Supplementary Figure 3: $\quad$ Multiple sequence alignments of $R$. seeberi 's sequence (AJ440719) with 16S rDNA in 11 cyanobacteria and cpDNA in flowering plant Carica papaya. Base numbering according to sequences available in NCBI nucleotide sequence database. Box shows sequence signature AATTTTCCG; stars indicate conserved bases; nucleotides flanking AATTTTCCG are identical in all the aligned sequences. Accession nos. are of following cyanobacteria: X59559.1 - Anabaena; AJ133161.1 - Nostoc; X84811.1 - O. agardhii; AF013028.1 - Trichodesmium; AF091109.1 Spirulina; X63`141.1 - Prochloron; AJ000708.1 - Cyanothece; U03403.1 - Microcystis; AF091110.1 - Plectonema; AB0150162.1 - Synechococcus; AB058219.1 - Phormidium; U76911.1 - chloroplast of Carica papaya; AJ440719 - Rhinosporidium seeberi

\begin{tabular}{|c|c|c|c|}
\hline \multirow{12}{*}{$\begin{array}{l}\text { X59559.1 } \\
\text { AJ133161.1 } \\
\text { X84811.1 } \\
\text { AF013028.1 } \\
\text { AF091109.1 } \\
\text { X63141.1 } \\
\text { AJ000708.1 } \\
\text { U03403.1 } \\
\text { AF091110.1 } \\
\text { ABO15062.1 } \\
\text { ABO50219.1 } \\
\text { U76911.1 } \\
\text { AJ440719.1 }\end{array}$} & \multirow{2}{*}{\multicolumn{3}{|c|}{$\begin{array}{l}119 \text { TTCAGGTCGGGGACAACCACTGGAAACGGTGGCTAATACCGGATGTGCCGAAAGGTGAAAGAT-TTATT-GCCTGAAGATGAGCTCGCGTCTGATTAGCT/ } \\
25 \text { TTCAGGTTCGGACAACCACTGGAACGGTGGCTAATACCGAATGTGCCGAGAGGTGAAGGC-TTGCT-GCCTGAAGATGAGCTCGCGTCTGATTAGCT/ }\end{array}$}} \\
\hline & & & \\
\hline & \multirow{2}{*}{\multicolumn{3}{|c|}{$\begin{array}{l}24 \text { TCTAGGACGGGGACAACAGTTGGAAACGACTGCTAAACCCGGATGAGCCGAAAGGTAAAAGAT-TAATC-GCCTAGAGAGGGGCTTGCGTCTGATTAGCTAGTTGGTGAGGTAAGAGCCC } 141 \\
92 \text { TTCAGGTCTGGGACAACAGAAGGAAACTTCTGCTAATCCCGGATGAGCCTTAGGGTAAAAGAT-AAATT-GCCTGGAGATGAGCTCGCGTCTGATTAGCTAGTTGGTGTGGTAAAGGCAT } 209\end{array}$}} \\
\hline & 92 TCTAGGTCGGGGACAACAGTTGGAAACGACTGCTAATCCCCGATGAGCCTAACGGTAAAAGAT-TTATT- GCCTAGAGAAGAGCTCGCGTCCGATTAGTTAGTTGGTGGGGTAAGAGCCT 209 & & \\
\hline & \multirow{2}{*}{\multicolumn{3}{|c|}{ 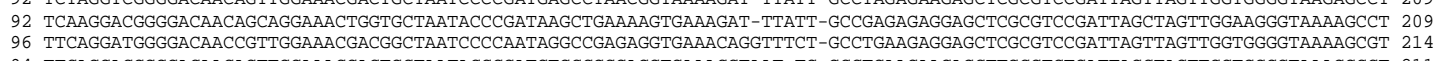 }} \\
\hline & & & \\
\hline & \\
\hline & \multirow{2}{*}{\multicolumn{3}{|c|}{$\begin{array}{l}94 \text { CAAAGGACGGGGACAACAGTGGGAAACCGCTGCTAAGACCCGATGTGCCGAGAGGTGAAACAT-TTATG-GCCTTTGGATGAACTCGCGTCTGATTAGCTAGTTGGTGTGGTAAGAGCAT } 211 \\
91 \text { TATAGGATGGGGACACAGGGAGAATTCTTGTTAACACCCGATGTGCCGAGAGGTGAAATAT-TAATA-GCCTGTAGATGAGCTCGCGTCTGATTAGCTAGTTGGTGGGGTAAGGCTT } 208\end{array}$}} \\
\hline & & & \\
\hline & \multicolumn{3}{|c|}{$\begin{array}{l}91 \text { TATAGGATGGGGACACAGGGAGAATTCTTGTTAACACCCGATGTGCCGAGAGGTGAAATAT-TAATA-GCCTGTAGATGAGCTCGCGTCTGATTAGCTAGTTGGTGGGGTAAAGGCTT } 208 \\
\text { O8 CTTAGGTGGGGACAACAGTTGGAAACGACTGCTAATACCGCATATGTCGAGAGATGAAAGAT-TTATT-GCCTGAGGATGAACTCGCGTCTGATTAGCTAGTAGGTGAGGTAAGAGCTT } 125\end{array}$} \\
\hline & \multicolumn{3}{|c|}{ 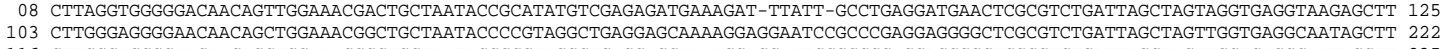 } \\
\hline & \multicolumn{3}{|c|}{ 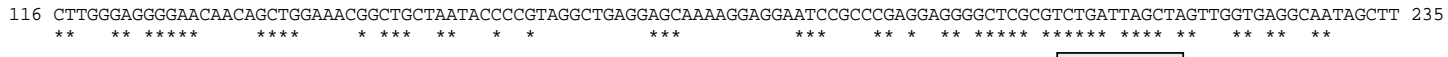 } \\
\hline & \multirow{13}{*}{ 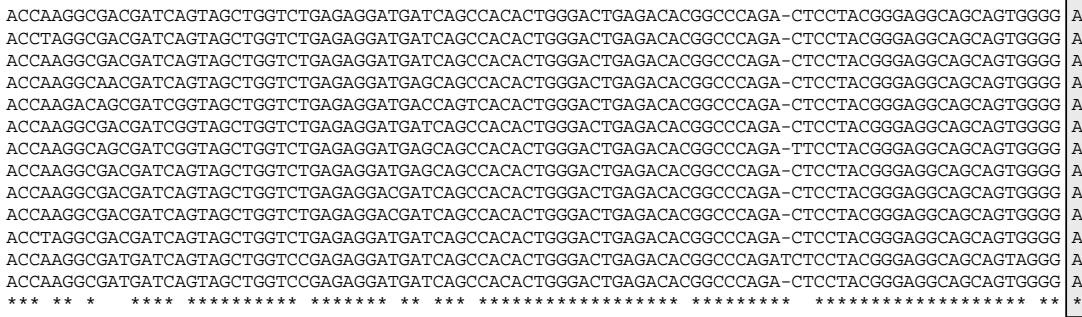 } & & \multirow[t]{2}{*}{ TGGGCGAAAGCCTG 3} \\
\hline & & & \\
\hline & & CGC & 10201 \\
\hline & & & \\
\hline & & & \\
\hline & & & \\
\hline & & & TG 3 \\
\hline & & AATTTTCCGC & AATGGGCGAAAGCCTG 330 \\
\hline & & & \\
\hline & & & \\
\hline & & & \\
\hline & & & \\
\hline & & & \\
\hline \multirow{2}{*}{\multicolumn{4}{|c|}{ 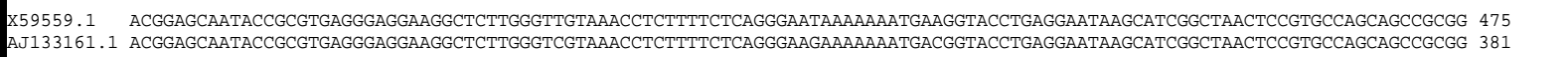 }} \\
\hline & \multirow{11}{*}{\multicolumn{3}{|c|}{ 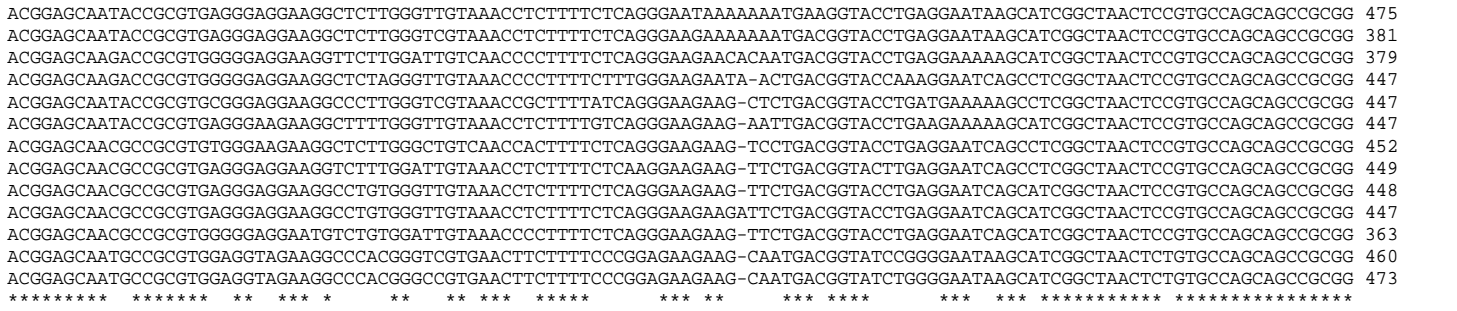 }} \\
\hline & & & \\
\hline & & & \\
\hline & & & \\
\hline & & & \\
\hline . & & & \\
\hline & & & \\
\hline & & & \\
\hline & & & \\
\hline & & & \\
\hline & & & \\
\hline & \\
\hline & \multicolumn{3}{|c|}{$\begin{array}{l}\text { TAATACGGAGGATGCAAGCGTTATCCGGAATGATTGGGCGTAAAGCGTCCG-CAGGTGGC TGTGTAAGTCTGCTGTTAAAGAGTCATGCTCAACATGATAAGAGCAGTGGAAACTACAC } \\
\text { TAGCGG }\end{array}$} \\
\hline & \multirow{2}{*}{\multicolumn{3}{|c|}{$\begin{array}{l}\text { TAATACGGGGGATGCAAGCGTTATCCGGAATGATTGGGGGTAAGAGTCCG-TAGGTAGT CATCCAAGTCTGCTGTTAAAGAGCGAGGCTTAACCTCGTAAAGGCAGTGGAACTGGAAG } 498 \\
\text { TAATACGGAGGAGGCAGCGTTATCCGGATGATTGGGCGTAAAGCGTCCG-CAGGTGGC CATGTAAGTCTGCTGTTAAAACCCAGGGCTTAACTCTGGTCAGGCAGTGGAAACTACAAA } 566\end{array}$}} \\
\hline & & & \\
\hline & \\
\hline & \multirow{2}{*}{\multicolumn{3}{|c|}{ 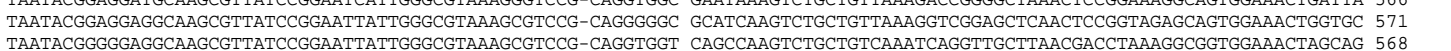 }} \\
\hline 0 & & & \\
\hline & \multicolumn{3}{|c|}{ TAAGACGGAGGATGCAAGCGTTATCCGGAATTATTGGGCGTAAAGCGTCCG-TAGGTGGT TATACAAGTCTGTTGTTAAAGCCCATAGCTCAACTGTGGATCGGCAATGGAAACTGTATG 567} \\
\hline & \multirow{2}{*}{\multicolumn{3}{|c|}{ 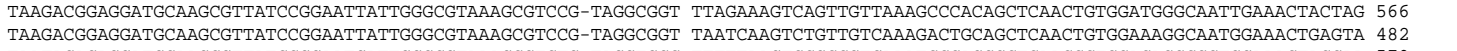 }} \\
\hline & & & \\
\hline & \multicolumn{3}{|c|}{$\begin{array}{l}\text { TAATACAGAGGATGCAAGCGTTATCCGGATGATTGGGGGTAAAGCGTCTG-TAGGTGGC TTTTTAAGTCCGCCGTCAAATCCCAGGGCTCAACCCTGGACAGGCGGTGGAAACTACCAA } 579 \\
\text { TAATACAGAGGATGCAAGCGTTATCCGGAATGATTGGGCGTAAAGCGTCTGGTAGGTGGC TTTTTAAGTCCGCCGTCAAATCTCAGGGCTCAACCCTGGACAGGCGGTGGAAACTACCAA 593 }\end{array}$} \\
\hline & & & \\
\hline
\end{tabular}


X59559.1

GCTAGAGTACGTTCGGGGCAGAGGGAATTCCTGGTGTAGCGGTGAAATGCGTAGAGATCA

GGAAGAACACCGGTGGCGAAAGCGCTCTGCTAGGCCGTAACTGACACTGAGGGACGAAAG 714

AJ133161.1

GCTAGAGTGCGTTCGGGGCAGAGGGATTCCTGGTGTAGCGGTGAAATGCGTAGAGATCA

GGAAGAACACCGGTGGCGAAAGCGCTCTGCTAGGCCGCAACTGACACTGAGGGACGAAAG 620

X84811.1

ACTAGAGTGTAGTAGGGGCAGAGGGATTCCTGGTGTAGCGGTGAAATGCGTAGAGATCA

GGAAGAACACCGGTGGCGAAGGCGCTCTGCTGGGCTATAACTGACACTGAGGGACGAAAG 618 AFO13028.1

GCTAGAGTTCGGTAGGGGCAAAGGGAATTCCCGGTGTAGCGGTGAAATGCGTAGATATCG

GGAAGAACATCGGTGGCGAAAGCGCTTTGCTAGACCAAAACTGACGCTCAGGGACGAAAG 686

AF०91109.1

ACTAGAGTACAGTAGGGGTAGAGGGAATTCCCAGTGTAGCGGTGAAATGCGTAGAGATTG

GGAAGAACACCGGTGGCGAAAGCGCTCTACTGGGCTGTAACTGACACTGAGGGACGAAAG 686

X63141.1

GCTAGAGTACGGTAGGGGTAGAGGGAATTCCCAGTGTAGCGGTGAAATGCGTAGATATTG

GGAAGAACACCGGTGGCGAAAGCGCTCTACTAGGCCGAAACTGACACTGAGGGACGAAAG 686

AJ०00708.1

GCTAGAGGGCGACAGGGGTAGAGGGAATTCCCAGTGTAGCGGTGAAATGCGTAGAGATTG

GGAAGAACACCGGTGGCGAAAGCGCTCTACTGGGTCGCACCTGACCCTCAGGGACGAAAG 691

U०3403.1 ACTAGAGAGCAGTAGGGGTAGCAGGAATTCCCAGTGTAGCG -

TGAAATGCGTAGAGATTG

GGAAGAACATCGGTGGCGAAAGCGTGCTACTGGGCTGTATCTGACACTCAGGGACGAAAG 687

AF०91110.1

ACTTGAGTGTGGTAGGGGTAGAGGGAATTCCTAGTGTAGCGGTGAAATGCGTAGATATTA

GGAAGAACACCAGTGGCGAAGGCGCTCTACTGGGCCATAACTGACACTGATGGACGAAAG 687

AB015062.1

ACTAGAGAGTGGTAGGGGTAGAGGGAATTCCTAGTGTAGCGGTGAAATGCGTAGATATTA

GGAAGAACACCAGTGGCGAAGGCGCTCTACTGGGCCATTACTGACGCTGATGGACGAAAG 686 AB०58219.1

ACTGGAGTGCGGTAGGGGTAGAGGGAATTCCCAGTGTAGCGGTGAAATGCGTAGATATTG

GGAAGAACACCAGTGGCGAAGGCGCTCTACTGGGCCGTAACTGACGCTGAGGGACGAAAG 602 U76911. 1

GCTGGAGTACGGTAGGGGCAGAGGGAATTTCCGGTGGAGCGGTGAAATGCGTAGAGATCG

GAAAGAACAGGAACGGCGAAAGCACTCTGCTGGGCCGACACTGACACTGAGAGACGAAAG 699

AJ440719.1

GCTGGAGTACGGTAGGGGCAGAGGGAATTTCCGGTGGGGCGGTGAAATGCGTAGAGATCG

GAAAGAACACCAACGGCGAAAGCACTCTGCTGGGCCGACACTGACACTGAGAGACGAAAG 713

$* * * * * * * * * * * * * * * * * * * * * * * * * * * * * * * * * * * * * *$

X59559. 1

CTAGGGGAGCGAATGGGATTAGATACCCCAGTAGTCCTAGCCGTAAACGATGGATACTAG GCGTGGCTTGTATCGACCCGAGCCGTGCCGGAGCCAACGCGTTAAGTATCCCGCCTGGGG 834 AJ133161.1

CTAGGGGAGCGAATGGGATTAGATACCCCAGTAGTCCTAGCCGTAAACGATGGATACTAG GCGTGGCTTGTATCGACCCGAGCCGTGCCGTAGCTAACGCGTTAAGTATCCCGCCTGGGG 740 X84811.1

CTAGGGGAGCGAATGGGATTAGATACCCCAGTAGTCCTAGCGGTAAACGATGGAAACTAG

GTGTGGCCTGTATCGACCCGGGCCGTGCCGAAGCAAACGCGTTAAGTTTCCCGCCTGGGG 738

AF013028.1

CTAGGGGAGCGAATGGGATTAGATACCCCAGTAGTCCTAGCCGTAAACGATGGATACTAG GTGTTGCCTGTATCGACCCAGGCAGTGCCGTAGCTAACGCGTTAAGTATCCCGCCTGGGG 806 AF091109.1

CTAGGGGAGCGAATGGGATTAGATACCCCAGTAGTCCTAGCCGTAAACGATGGAAACTAG

GCGTGGCTTGTATCGACCCGAGCCGTGCCGAASSTAACGCGTTAAGTTTCCCGCCTGGGG 806

X63141.1

CTAGGGTAGCGAAAGGGATTAGATACCCCTGTAGTCTTAGCGGTAAACGATGGATACTAG GTGTAGCTTGTATCGACCCGAGCTGTGCCGAAGCTAACGCGATAAGTATCCCGCCTGGGG 806 
X59559.1 CCAGC-ATTAAGTTGGGCACTCTAGAGAGACTGCCGGTGACAAACCGGAGGAAGGTGGGG ATGACGTCAAGTCAGCATGCCCCTTACGTCTTGGGCTACACACGTACTACAATGCTACGG 1192

AJ133161. 1 CCATC -ATTAAGTTGGGCACTCTAGAGAGACTGCCGGTGACAAACCGGAGGAAGGTGGGG ATGACGTCAAGTCAGCATGCCCCTTACGTCTTGGGCTACACACGTACTACAATGCTCCGG 1098

X84811.1 CCATC-ATTAAGTTGGGAACTCTAGCGAGACTGCCGGTGACAAACCGGAGGAAGGTGAGG ATGACGTCAAGTCAGCATGGCCCTTACGTCCTGGGCGACACACGTACTACAATGCGAGGG 1096

AF013028.1 CCATC -ATTAAGTTGGGCACTCTGGAGAGACTGCCGGTGACAAACCGGAGGAAGGTGGGG ATGACGTCAAGTCAGCATGCCCCTTACGCTCTGGGCTACACACGTACTACAATGGTTGGG 1164 AF091109. 1 CTAGCGATTTAATCGCGCACTCTAGGGAGACTGCCGGGGAAAACTCGGAGGAAGGTGGGG ATGACGTCAAGTCAGCATGCCCCTTACGTCCTGGGCTACACACGTACTACAATGGTCGAG 1165

X63141.1 CCAAT-ATTAAGTTAGGCACTCTAGGGAGACTGCCGGGGACAACTCGGAGGAAGGTGGGG ATGACGTCAAGTCAGCATGCCCCTTACGTCCTGGGCGACACACGTACTACAATGGTTGGG 1164

AJ000708.1 CCAGC-ATTAGGTTGGGCACTCTAGGGAGACTGCCGGTGACAAACCGGAGGAAGGTGAGG ATGACGTCAAGTCAGCATGCCCCTTATGCTCTGGGCAACACACGTCCTACAATGGTCGGG 1169 U03403.1 CCAGC - ATTAAGTTGGGGACTCTAAGGAGACTGCCGGTGACAAACCGGAGGAAGGTGGGG ATGACGTCAAGTCAGCATGCCCCTTACGTCTTGGGCGACACACGTACTACAATGGTCGGG 1164 AF091110.1 CCATC - ATTAAGTTGGGCACTTTGAGGAGACTGCCGGTGACAAACCGGAGGAAGGTGGGG ATGACGTCAAGTCATCATGCCCCTTACATCCTGGGCTACACACGTACTACAATGCAACGG 1165

AB015062.1 CCATC-ATTAAGTTGGGCACTTTAAGGAGACTGCCGGTGACAAACCGGAGGAAGGTGGGG ATGACGTCAAGTCATCATGCCCCTTACATCCTGGGCTACACACGTACTACAATGCAACGG 1164 AB058219.1 CCATC - ATTAAGTTGGGCACTCTGGGGAGACTGCCGGGGACAACTCGGAGGAAGGTGTGG ATGACGTCAAGTCATCATGCCCCTTACGTTCTGGGCTACACACGTACTACAATGCTTCGG 1080 U76911.1 CCACC-GTTGAGTTTGGAACCCTGAGCAGACTGCCGGTGATAAGCCGGAGGAAGGTGAGG ATGACGTCAAGTCATCATGCCCCTTATGCCCTGGGCGACACACGTGCTACAATGGCCGGG 1177 AJ440719. 1 CCACC - GTTGAGTTTGGAACCCTGAACAGACTGCCGGTGATAAGCCGGAGGAAGGTGAGG ATGACGTCAAGTCATCATGCCCCTTATGCCCTGGGCGACACACGTGCTACAATGGCCGGG 1192

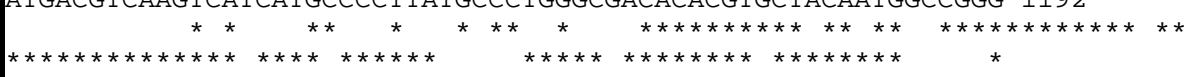

X59559.1 ACAGAGGGCAGCAAGCTAGCGATAGCAAGCAAATCCCG-TAAACCGTAGCTCAGTTCAGA TCGCAGGCTGCAACTCGCCTGCGTGAAGGAGGAATCGCTAGTAATTGCAGGTCAGC - ATA 1310

AJ133161.1 ACAAAGGGCAGCAAGCTAGTGATAGCAAGCAAATCCCA-TAAACCGGAGCTCAGTTCAGA TCGAAGGCTGCAACTCGCCTTCGTGAAGTCGGAATCGCTAGTAATTGCAGGTCAGC - ATA 1216

X84811.1 ACAGAGAGCAGCCAACCCGCGAGGGAGAGCGAATCTCA-TAAACCCTGGCACAGTTCAGA TTGCAGGCTGCAACTCGCCTGCATGAAGGAGGAATCGCTAGTAATCGGAGGTCAGC - ATA 1214

AF013028.1 ACAGAGGGCAGCGAACCCGAGAGGGGAAGCCAATCCCC - AAAACCCAGCCTCAGTTCAGA TTGCAGGCTGCAACTCGCCTGCATGAAGGAGGAATCGCTAGTAATCGCAGGTCAGC - ATA 1282

AF091109.1 ACAAAGGGCAGCAAACTGGCAACAGCAAGCGAATCCCAGCAAACTCGGTCACAGTTCGGA TTGCAGGCTGAAACTCGCCTGCATGAAGGAGGAATCGCTAGTAATCGCAGGTCAGC - ATA 1284

X63141.1 ACAAAGGGCAGCAAACTCGCGAGAGTAAGCGAATCTCATCAAACCCAGCCACAGTTCAGA TTGCAGGCTGCAACTCGCCTGCATGAAGTAGGAATCGCTAGTAATCGCAGGTCAGC - ATA 1283

AJ000708.1 ACAAAGGGGAGCAAGCTCGCGAGAGTAAGCGAATCCCAGCAAACTCGGCCTCAGTTCAGA TAGCAGGCTGCAACTCGCCTGCTTGAAGGAGGAATCGCTAGTAATCGCAGGTCAGA-ATA 1288 U03403.1 ACAAAGGGCAGCGAACTCGCGAGAGCCAGCGAATCCCAGCAAACCCGGCCTCAGTTCAGA TTGCAGGCTGCAACTCGCCTGCATGAAGGAGGAATCGCTAGTAATCGCCGGTCAGC - ATA 1283 AF○91110.1 ACAAAGGGCAGCCAACTAGCGATAGTGCGCTAATCCCA-TAAACCGTTGCTCAGTTCAGA TTGCAGGCTGCAACTCGCCTGCATGAAGGCGGAATCGCTAGTAATCGCAGGTCAGC - ATA 1283

AB015062.1 ACAAAGGGCAGCCAACTAGCGATAGTGAGCTAATCCCA-TAAACCGTTGCTCAGTTCAAA TTGCAGGCTGCAACTCGCCTGCATGAAGGCGGAATCGCTAGTAATCGCAGGTCAGC - ATA 1282 AB058219.1 ACAAAGGGCAGCAAGCGTGCGAGCGCAAGCTAATCCCA-TAAACCGAGGCACAGTTCAGA TTGCAGGCTGCAACTCGCCTGCATGAAGGAGGAATCGCTAGTAATCGCAGGTCAGC - ATA 1198 U76911.1 ACAAAGGGTCGCGATCCCGCGAGGGTGAGCTAACTCCA-AAAACCCGTCCTCAGTTCGGA TTGCAGGCTGCAACTCGCCTGCATGAAGCCGGAATCGCTAGTAATCGCCGGTCAGCCATA 1296 AJ440719.1 ACAAAGGGTCGCGATCCCGCGAGGGTGAGCTAACTCCA-AAAACCCGTCCTCAGTTCGGA TTGCAGGCTGCAACTCGCCTGCATGAAGCCGGAATCGCTAGTAATCGCCGGTCAGCCATA 1311

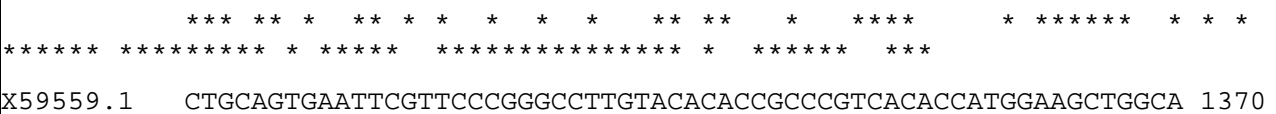

